\title{
Materials on the nanoscale: Total scattering analysis for nanoparticle chemistry K. M. Jensen ${ }^{1}$ \\ ${ }^{1}$ No affiliation given \\ kirsten@chem.ku.dk
}

Nanomaterials have come to play a huge role in modern materials chemistry: By nanosizing the functional materials used in a range of different applications, the properties of the materials can be improved, and new applications can arise. This development has challenged the conventional techniques for material characterization. However, total scattering combined with Pair Distribution Function analysis allows us to look further into nanostructure and establish the structure-property relation for advanced functional materials.1 Here, I will present recent work illustrating how we use x-ray total scattering to study atomic structure in advanced nanomaterials, with special focus on transition metal oxide nanoparticles. We observe that new structural motifs, unstable in the bulk form, become dominant in nanoscale materials. 2

Apart from studying the synthesized nanoparticles, I will also show how in situ x-ray total scattering allows following the formation of materials. Despite decades of research into nucleation processes, very little is known on how nanoparticle formation during solvothermal synthesis takes places on the atomic scale. We have developed methods which allows using in situ synchrotron X-ray Total Scattering and Pair Distribution Function analysis to follow nanoparticle nucleation and growth in situ. 3 In contrast to conventional crystallographic studies, PDF analysis gives structural information from non-crystalline species, allowing obtaining structural information on the atomic scale, all the way from precursor to the final nanoclusters during synthesis. Using x-ray total scattering combined with small angle X-ray scattering, we deduce the atomic structure of prenucleation clusters, present in the processes just before the crystalline nanoparticles have formed. We show that the solvent and synthesis conditions have large influence on the nucleation pathway and the structure of the nano-scale clusters in the synthetic pathway.

1. Christiansen, T. L.; Cooper, S. R.; Jensen, K. M. Ø. There's no place like real-space: elucidating size-dependent atomic structure of nanomaterials using pair distribution function analysis. Nanoscale Adv. 2020, 2 (6), 2234-2254.

2. Christiansen, T. L.; Bøjesen, E. D.; Juelsholt, M.; Etheridge, J.; Jensen, K. M. Ø. Size Induced Structural Changes in Molybdenum Oxide Nanoparticles. ACS Nano 2019, 13 (8), 8725-8735.

3. Juelsholt, M.; Lindahl Christiansen, T.; Jensen, K. M. Ø. Mechanisms for Tungsten Oxide Nanoparticle Formation in Solvothermal Synthesis: From Polyoxometalates to Crystalline Materials. J. Phys. Chem. C 2019, 123 (8), $5110-5119$.

4. Aalling-Frederiksen, O.; Juelsholt, M.; Anker, A. S; Jensen, K. M. Ø. Formation and growth mechanism for niobium oxide nanoparticles: Atomistic insight from in situ X-ray total scattering, Nanoscale, 2021, 13 (17), 8087-8097, 